\title{
Paralinguistic Processing in Children with Callosal Agenesis : Emergence of Neurolinguistic Deficits
}

\author{
Warren S. Brown ${ }^{1}$, Melissa Symingtion ${ }^{1}$, Diana VanLancker-Sidtis ${ }^{2}$, \\ Rosalind Dietrich ${ }^{3}$, and Lynn K. Paul ${ }^{1}$
}

1. The Travis Research Institute, Center for Biopsychosocial Research, Fuller Graduate School of Psychology

2. Dept. of Speech-Language Pathology and Audiology, Steinhardt School of Education, New York University

3. Dept. of Radiology, School of Medicine, University of California, Irvine

Running Title: $\quad$ Paralinguistics in Children with ACC

Corresponding Author: Warren S. Brown, Ph.D.

The Travis Research Institute

180 N. Oakland Ave.

Pasadena, CA 91101

U.S.A.

wsbrown@fuller.edu 


\begin{abstract}
Recent research revealed impaired processing of both nonliteral meaning and affective prosody in normally intelligent adults with agenesis of the corpus callosum (ACC). Since normal children have incomplete mylenization of the corpus callosum, it was hypothesized that paralanguage deficits in children with ACC would be less apparent relative to their peers. The Familiar and Novel Language Comprehension test (FANL-C) and Prosody Test were given to 18 acallosal children with normal intelligence and 17 controls matched for age $(7-13$ years $)$, education, and IQ $(83-122)$. When controlling for age, children with ACC were significantly poorer in comprehension of the precise meaning of both literal and nonliteral items on the FANL-C. Adults with ACC had previously been shown to have difficulty only on nonliteral items. The effect size for nonliteral comprehension in children with ACC was smaller than that seen in adults. There was only a trend for the child ACC group to perform worse on the recognition of affective prosody. Thus, while deficits in paralinguistic processing were apparent, children with ACC were not as clearly different from age peers as adults, and were equally deficient at comprehending literal expressions. The differences in results between adults and children with ACC are thought to reflect incomplete callosal development in normal children, and the importance of the corpus callosum in the early stages of the development of the ability to process literal language.
\end{abstract}

Key Words: Corpus callosum, agenesis of the corpus callosum, nonliteral language, idioms, prosody, child development 


\section{Introduction}

The study of children with agenesis of the corpus callosum (ACC) who have normal-range IQs promises to provide insight into the specific role of the corpus callosum in the development of neuropsychological abilities. While many children with ACC are mentally retarded, there exists a large group of individuals with ACC who have normal intelligence and few, if any, other neurological abnormalities or disorders.

The most consistent domain of neuropsychological deficit in individuals who have ACC and normal intelligence seems to be complex novel problem solving(Brown \& Paul, 2000; Schieffer, Paul, \& Brown, 1998). These results lead to the generalization that individuals with ACC have deficits in fluid intelligence, even when crystallized aspects of intelligence are normal.

A few studies have focused on basic language processing in individuals with ACC, with various subtle deficits having been described with respect to phonemic recognition (Temple, Jeeves, \& Vilarroya, 1989, 1990) and syntactic processing (Dennis, 1981; Sanders, 1989). However, families of normally intelligent children and adolescents with ACC report that they have marked difficulties with the social and pragmatic aspects of language, but no apparent problems in basic language skills. Thus, development of paralinguistic skills may be more susceptible to callosal absence than other aspects of language.

Consistent with the observations of family members, we recently reported data from adults with ACC and normal IQ demonstrating significant deficits in paralinguistic processing (Paul, Van Lancker-Sidtis, Schieffer, Dietrich, \& Brown, 2003). Adults with ACC had normal ability to understand literal expressions, but significant deficits in 
comprehension of nonliteral expressions. In addition, adults with ACC had difficulty understanding affective prosody, and a deficiency in the comprehension of proverbs (as indicated in both multiple choice and free-answer response formats). While these deficits are clear in adults with ACC, the level of deficit in either the appreciation of prosody or the understanding of nonliteral language in children with ACC has not previously been studied.

Integration into language comprehension and expression of right-lateralized linguistic processing would appear to depend on interhemispheric interactions. There is evidence to suggest that the processing of nonliteral phrases such as idioms is particularly affected by dysfunction of the right cerebral hemisphere (Benton, 1968; Brownell, Potter, Bihrle, \& Gardner, 1986; Kempler, Van Lancker, Marchman, \& Bates, 1999; Van Lancker \& Kempler, 1987; Weylman, Brownell, Roman, \& Gardner, 1989; Winner \& Gardner, 1977). A unique role of the right (nondominant) hemisphere in processing prosody and emotional speech has also been reported (Bowers, Bauer, \& Heilman, 1993; Heilman, Scholes, \& Watson, 1975; Starkstein, Federoff, Price, Leiguarda, \& Robinson, 1994; Tucker, 1981; Weintraub \& Meulam, 1983). Other studies suggest that the left and right hemispheres play complementary roles in processing of the prosodic aspects of language (Pell \& Baum, 1997; Robin, Tranel, \& Damasio, 1990; Zatorre, Evans, Meyer, \& Gjedde, 1992). A role for interhemispheric pathways in the production and comprehension of affective prosody has been suggested by several studies (Cancelliere \& Kertesz, 1990; Van Lancker \& Breitenstein, in press; Van Lancker, Pachana, Cummings, Sidtis, \& Erickson, 1996). 
In normal children, the corpus callosum is not fully myelinated until sometime during the early years of the second decade of life (Giedd et al., 1994; Yakovlev \& Lecours, 1967). Increased myelinization of the corpus callosum would contribute to the speed and efficiency of interhemispheric interactions. Similarly, paralinguistic skills in normal children have also been found to develop between approximately 5 and 10 years old (Cohen, Prather, Town, \& Hynd, 1990; Kempler et al., 1999). The deficits in paralinguistic process in adults with ACC which we previously demonstrated (Paul et al., 2003) suggests that callosal development may be important for the emergence of the comprehension of nonliteral language and prosody.

This report is a follow-up on our study of adults with ACC (Paul et al., 2003). We describe linguistic processing in children with ACC with respect to comprehension of both literal and nonliteral expressions, and recognition of affective prosody. While deficits similar to those found in adults with ACC were expected in children with ACC, given the lack of complete development of both the corpus callosum or paralinguistic processing ability in normal children, it was hypothesized that the degree of difference in performance between normal children and children with ACC would be less than that seen in adults.

\section{Methods}

\section{Participants}

Participants included 18 children (ages 7 to13; mean age $=9.3$ ) with complete ACC and IQs in the borderline to high average range (FSIQ 83 to122, mean $=91.0$ ). Magnetic resonance images (MRIs; a CT scan in one case) and neuroradiological reports 
were obtained for all individuals with ACC. The diagnosis of ACC was confirmed by a second neuroradiologist, who inspected scans for presence or absence of the CC and anterior commissure, and for any evidence of other brain malformations.

Control participants included 17 children matched for age (ages 8 to 12 ; mean $=$ 9.6), IQ (FSIQ 83 to 120 , mean $=96.8$ ), and sex. Control participants were recruited from two local private elementary schools. There were no significant differences in age $(\mathrm{t}=0.62, \mathrm{~ns})$ or IQ $(\mathrm{t}=1.86, \mathrm{p}=.074)$ between the ACC and control groups.

Exclusionary criteria for children with ACC included MRI evidence of major neuropathology in addition to ACC. For both groups of children exclusionary criteria also included: English as a second language, IQ less than 80, history of major head trauma, neurosurgery, other CNS disease, seizure history (more than 2 seizures), psychopathology, or current psychotropic medication that would interfere with test performance.

Parents signed consent forms for participation of their children. All participants were treated in accordance with the American Psychological Association Ethical Principles (American Psychological Association, 1992) throughout the experiment. Research procedures were approved by the Human Subject Review Committee at the Fuller Graduate School of Psychology, and also, for testing normal children, by school administrative personnel.

\section{Materials}

The Familiar and Novel Language Comprehension Test (FANL-C) was used to examine comprehension of literal and nonliteral language. The FANL-C (Kempler \& Van Lancker, 1985) is a test of 40 statements, 20 literal and 20 nonliteral expressions, 
matched for word length, lexical complexity, and grammatical structure. Each statement is paired with four cartoon drawings, and the participant is asked to point to the picture that best matches the statement. The number of Literal items correctly identified reflected the participant's ability to interpret concrete meanings of the sentences. Likewise, the number of Nonliteral statements correctly identified measures the ability of the participant's understanding of common, nonliteral, idiomatic phrases.

The Prosody Test is a 16-item test created by Van Lancker (Van Lancker, 1988) to assess ability to identify affective prosody apart from sentence content. Subjects are asked to listen to a tape on which simple sentences are read with 4 types of emotional prosody (surprise, angry, happy, sad). The labels typically associated with these emotional tones were not mentioned to the patients. They were then asked to "point to the picture of the face which displays the emotion expressed by the reader."

Due to the ages of the children in this study, the Proverbs Tests were not administered as they were in our study of adults with ACC.

\section{Results}

Means and standard deviations for outcome measures can be found in Table 1. Since paralinguistic skills are developing normally during this developmental period and are presumably affected by intelligence, age and FSIQ were used as covariates in all statistical tests. Age was found to be a significant covariate with respect to interpretation

of both literal $(F=5.89, d f=1,31 . \mathrm{p}<.021)$ and nonliteral $(F=24.82, d f=1,31, p<.001)$ statements, but was not significant for affective prosody $(F=1.04, d f=1,25, \mathrm{~ns})$. FSIQ was a significant covariate for comprehension of nonliteral statements $(F=5.22, d f=$ 
1,31. $p<.029)$, but was not significant for either literal statements $(F=0.35, d f=1,31$, ns) or affective prosody $(F=0.47, d f=1,25, \mathrm{~ns})$.

Prosody Test: One control and 5 individuals with ACC were not administered this version of prosody test due to a change in our testing protocol. For the remainder of participants, children with ACC performed more poorly than controls. Statistical comparison resulted in a nonsignificant trend $(F=2.86, d f=1,25, p=.10)$.

FANL-C: An ANOVA (group-by-subtest controlling for age) showed a significant difference in performance on the Literal vs. Nonliteral subtests $(F=27.05, d f=1,31, p<$ .001) such that both groups performed better on the literal items. Also, individuals with ACC performed significantly worse than controls $(F=8.23, d f=1,31, p<.01)$.

However, the group-by-subtest interaction was not significant $(F=0.47, d f=1,31, \mathrm{~ns})$. Post hoc comparison by group showed a significant difference between the groups on both the literal $(F=5.08, d f, 1,31, p<.05)$ and nonliteral subscales $(F=4.74, d f, 1,31$, $p<.05)$ of the FANL-C.

\section{Discussion}

These results demonstrate that children with ACC have significantly greater difficulty than controls in understanding both literal and nonliteral language, as well as suggesting that they also may have difficulty in comprehending affective prosody. This outcome is generally consistent with the previous study of adults with ACC in that adults with ACC were found to have deficits in the comprehension of nonliteral language and affective prosody (Paul et al., 2003). However, there were several important differences in the results from adults and children with ACC. 
First, on the FANL-C children with ACC were found to have as much difficulty in the processing of literal as nonliteral language. That is, the interaction between subtest and group was not significant, and the post-hoc tests for group differences on each subtest indicated nearly equivalent effect sizes $\left(\dot{\eta}_{\mathrm{p}}^{2}\right.$ [partial Eta squared] $=.141$ and .133 for literal and nonliteral, respectively). In contrast, adults with ACC performed similar to controls on literal items, but were deficient on nonliteral items, indexed by very different effect sizes for comparisons with controls $\left(\dot{\eta}_{\mathrm{p}}^{2}=.002\right.$ for literal and .318 for nonliteral).

With respect to the literal items, we suspect that this difference in outcome may reflect the difficulties experience by individuals with ACC in novel complex problem solving, i.e. difficulty in any domain of cognitive processing that has not yet become automatized. For children with ACC, comprehension of literal language may still be a task requiring some amount of problem solving, but is no longer so for normal children.

Effect sizes for nonliteral items suggest a second difference between the outcomes of our previous study of acallosal adults versus this study of children with ACC. The gap in performance between individuals with ACC and controls was greater for adults $\left(\dot{\eta}_{\mathrm{p}}^{2}=\right.$ .318) than for children $\left(\dot{\eta}_{\mathrm{p}}^{2}=.133\right)$. Similarly, differences between the groups were reduced in children on the test of understanding affective prosody $\left(\dot{\eta}_{\mathrm{p}}^{2}=.243\right.$ for adults and .103 for children). This reduced size of deficit in the comprehension of nonliteral language and affective prosody for children with ACC compared to adults may reflect differences in their relationship to their respective normal control groups. That is, the corpus callosum is known to continue the process of myelinization well into the second decade of life (Yakovlev \& Lecours, 1967; Giedd et al., 1996). Therefore, the normal children in this study cannot be presumed to have a corpus callosum that functions in a 
manner similar to that of normal adults. Thus, the deficit found in those with ACC would not be expected to be as large when compared to normal children who do not yet have a completely myelinated corpus callosum. These results imply that relative deficits in cognitive and linguistic abilities in individuals with ACC will emerge developmentally as the corpus callosum of their peers becomes more completely functional.

In general, the effects of callosal absence on neurocognitive performance can be understood using two complementary theories. First, deficits in ACC can be accounted for by reduced overall neural connectivity, such that the neural processing mass that is available for ongoing cognitive processing and problem solving is reduced. According to this theory, problem solving in areas that are relatively complex and novel (and thus not yet automated) demands larger cortical networks, including interhemispheric interactions, for normal processing speed and efficiency. Thus, ACC reduces the size of neural networks that are available for cognitive processing. For the children in this study with a normal corpus callosum, understanding literal and nonliteral expressions, as well as recognition of affective prosody, can engage interhemispheric processing networks not available to children with ACC.

A second theory by which one can understand deficits in individuals with ACC is related to hemispheric specialization. Here ACC would presumably result in some degree of isolation of right hemisphere processing from left hemisphere language systems. Given the literature suggesting that processing of both nonliteral language (Benton, 1968; Brownell et al., 1986; Kempler et al., 1999; Van Lancker \& Kempler, 1987; Weylman et al., 1989; Winner \& Gardner, 1977) and affective prosody (Bowers et al., 1993; Heilman et al., 1975; Starkstein et al., 1994; Tucker, 1981; Weintraub \& 
Meulam, 1983) may be localized to some degree in the right hemisphere, this theory can explain the results for adults with ACC, and at least some of the results for children. However, the significant deficit in ACC children in the processing of both literal and nonliteral expressions on the FANL-C is better understood from the point of view of reduced size of available cortical processing networks.

Alternatively, the theory might be advanced that the deficits demonstrated herein were not specifically related to ACC but were caused by other neuropathology present in a few of the children with ACC. Within our ACC group only 3 children had other abnormalities identified in the MRI, of whom 1 was taking Zoloft. However, these subjects did not appear to account for the deficient performance of the ACC group. Only 1 of these 3 children was among the bottom 3 subjects for scores on any of the tasks. In summary, the results presented herein demonstrate deficits in children with ACC in paralinguistic information processing involved in the understanding of nonliteral language, as well as a suggestion of a similar problem in the comprehension of affective prosody. In contrast to acallosal adults, children with ACC also had deficits in the understanding of literal expressions. Taken together, these results demonstrate that callosal absence has an impact on the comprehension of the meaning of language at several levels, even when IQ is normal. However, evidence of these deficits may emerge more clearly as the corpus callosum of age-peers becomes increasingly functional. 


\section{References}

Benton, A. (1968). Differential behavioral effects in frontal lobe disease. Neuropsychologia, 6, 53 - 60.

Bowers, D., Bauer, R. M., \& Heilman, K. M. (1993). The nonverbal affect lexicon: Theoretical perspectives from neurological studies of affect perception. Neuropsychology, 7, 422 - 444.

Brown, W. S., \& Paul, L. K. (2000). Psychosocial deficits in agenesis of the corpus callosum with normal intelligence. Cognitive Neuropsychiatry, 5.

Brownell, H., Potter, H., Bihrle, A., \& Gardner, H. (1986). Inference deficits in right brain-damaged patients. Brain and Language, 27, 310-321.

Cancelliere, A., \& Kertesz, A. (1990). Lesion localization in acquired deficits of emotional expression and comprehension. Brain and Cognition, 13, 133 - 147.

Cohen, M., Prather, A., Town, P., \& Hynd, G. (1990). Neurodevelopmental differences in emotional prosody in normal children and children with left and right temporal lobe epilepsy. Brain and language., 38(1), 122-134.

Dennis, M. (1981). Language in a congenitally acallosal brain. Brain and Language, 12, $33-53$.

Giedd, J. N., Castellansos, F. X., Casey, B. J., Kozuch, P., King, A. C., Hamburger, S. D., et al. (1994). Quantitative morphology of the corpus callosum in attention deficit hyperactivity disorder. American Journal of Psychiatry, 151, 665-669.

Heilman, K. M., Scholes, R., \& Watson, R. T. (1975). Auditory affective agnosia. Journal of Neurology, Neurosurgery, and Psychiatry, 38, 69 - 72. 
Kempler, D., \& Van Lancker, D. (1985). The Familiar and Novel Language Comprehension Test (FANL-C): Unpublished manuscript.

Kempler, D., Van Lancker, D., Marchman, V., \& Bates, E. (1999). Idiom comprehension in children and adults with unilateral brain damage. Developmental Neuropsychology, 15, 327-349.

Paul, L. K., Van Lancker-Sidtis, D., Schieffer, B., Dietrich, R., \& Brown, W. S. (2003). Communicative deficits in agenesis of the corpus callosum: nonliteral language and affective prosody. Brain and language., 85(2), 313-324.

Pell, M. D., \& Baum, S. R. (1997). Unilateral brain damage, prosodic comprehension deficits, and the acoustic cues to prosody. Brain and Language, 57, 195 - 214.

Robin, D. A., Tranel, D., \& Damasio, H. (1990). Auditory perception of temporal and spectral events in patients with focal left and right cerebral lesions. Brain and Language, 39, 539 - 555.

Sanders, R. J. (1989). Sentence comprehension following agenesis of the corpus callosum. Brain and Language, 37, 59-72.

Schieffer, B., Paul, L. K., \& Brown, W. S. (1998). Deficits in complex problem solving in agenesis of the corpus callosum (Abstract). Journal of the International Neuropsychological Society, 4, 20.

Starkstein, S. E., Federoff, J. P., Price, T. R., Leiguarda, R. C., \& Robinson, R. G. (1994). Neuropsychological and neuroradiologic correlates of emotional prosody comprehension. Neurology, 44, 515 - 522.

Temple, C. M., Jeeves, M. A., \& Vilarroya, O. (1989). Ten pen men: Rhyming skills in two children with callosal agenesis. Brain and Language, 37, 548-564. 
Temple, C. M., Jeeves, M. A., \& Vilarroya, O. (1990). Reading in callosal agenesis. Brain and Language, 39, 235-253.

Tucker, D. M. (1981). Lateralized brain function, emotion speech: Neurolinguistic studies. Psychological Bulletin, 89, 19 - 46.

Van Lancker, D. (1988). Heterogeneity in language and speech: Neurolinguistic studies. In A. Ellis (Ed.), Progress in the Psychology of Language (pp. 25 - 32). Hillsdale, NJ: Lawrence Erlbaum.

Van Lancker, D., \& Breitenstein, C. (in press). Emotional dysprosody and similar dysfunctions. In J. Bougousslavsky \& J. L. Cummings (Eds.), Disorders of behavior and mood in focal brain lesions. Cambridge, UK: Cambridge University Press.

Van Lancker, D., \& Kempler, D. (1987). Comprehension of familiar phrases by left- but not right-hemisphere damaged patients. Brain and Language, 32, 265 - 277.

Van Lancker, D., Pachana, N., Cummings, J., Sidtis, J., \& Erickson, C. (1996). Dysprosodic speech following basal ganglia stroke: Role of frontosubcortical circuits. Journal of the International Neuropsychological Society, 2, 5.

Weintraub, S., \& Meulam, M. M. (1983). Developmental learning disabilities and the right hemisphere: Emotional, interpersonal and cognitive components. Archives of Neurology, 40, $463-468$.

Weylman, S. T., Brownell, H. H., Roman, M., \& Gardner, H. (1989). Appreciation of indirect requests by left and right brain-damaged patients: The effects of verbal context and conventionality of wording. Brain and Language, 36, $580-591$. 
Winner, E., \& Gardner, H. (1977). The comprehension of metaphor in brain-damaged patients. Brain, 100, $717-729$.

Yakovlev, P. I., \& Lecours, A. R. (1967). The myelogenetic cycles of regional naturation in the brain. In A. Minowski (Ed.), Regional development of the brain in early life (pp. 98-114). Oxford: Blackwell.

Zatorre, R. J., Evans, A., Meyer, E., \& Gjedde, A. (1992). Lateralization of phonetic and pitch discrimination in speech processing. Science, 256, $846-849$. 
Paralinguistic in Children with ACC

Table 1

Mean FANL-C (Literal and Nonliteral subscales) and Prosody Scores ( \pm 1 standard deviation) for Child Controls and Children with ACC

FANL-C

Group

ACC Children 18

$19.94( \pm 1.96) * *$

$6.11( \pm 3.95)^{* *}$

$9.18( \pm 3.59)$

Child Controls $17 \quad 18.29( \pm 1.45)$
Prosody

N Prosody

$\begin{array}{llllll}\text { ACC Children } & 18 & 19.94( \pm 1.96)^{* *} & 6.11( \pm 3.95)^{* *} & 13 & 11.0( \pm 4.00)^{*} \\ \text { Child Controls } & 17 & 18.29( \pm 1.45) & 9.18( \pm 3.59) & 16 & 13.25( \pm 2.82)\end{array}$

* Difference from controls, $\mathrm{p}=.10$.

** Difference from controls, $\mathrm{p}<.05$. 